\section{FRI0710-HPR EFFECTIVENESS OF EXERCISE IN THE MANAGEMENT OF FATIGUE AND SLEEP QUALITY IN FIBROMYALGIA: A SYSTEMATIC REVIEW AND META-ANALYSIS}

Fernando Estevez-Lopez ${ }^{1,2}$, Cristina Maestre Cascales ${ }^{3}$, Deborrah Russell ${ }^{1}$, Inmaculada C. Alvarez-Gallardo ${ }^{4}$, María Rodriguez-Ayllon ${ }^{2}$, Ciara Hughes ${ }^{1}$, Gareth W. Davison ${ }^{1}$, Borja Sañudo ${ }^{5}$, Joseph Mcveigh ${ }^{6} .{ }^{1}$ Ulster University, Belfast, United Kingdom; ${ }^{2}$ University of Granada, Granada, Spain; ${ }^{3}$ Universidad Politécnica de Madrid, Madrid, Spain; ${ }^{4}$ University of Cadiz, Cadiz, Spain; ${ }^{5}$ University of Seville, Seville, Spain; ${ }^{6}$ University College Cork, Cork, Ireland

Background: Non-pharmacological interventions are the mainstay of treatment for fibromyalgia, however, current evidence-based guidelines report that the only therapy supported by 'strong for' evidence is exercise intervention for pain in fibromyalgia [1]. While increased fatigue and poor sleep quality are among the most burdensome symptoms in fibromyalgia, there remains limited evidence for the effectiveness of exercise in the management of these symptoms [2,3]

Objectives: To determine the effectiveness of exercise in the management of fatigue and sleep quality in fibromyalgia.

Methods: A systematic search was conducted using PubMed and Web of Science in October 2018 (Prospero Registration No. CRD42018118005). Eligible studies were randomised controlled trials (RCT) including adults with fibromyalgia (population), who received exercise (intervention) compared to usual care (comparator). Outcomes of interest were fatigue and/ or sleep quality. No restrictions were applied for language nor for publication date. Random effects meta-analyses were conducted. The Cochrane Collaboration's tool was used for assessing risk of bias in the included studies. The quality of evidence was evaluated using the Grading of Recommendations Assessment, Development, and Evaluation (GRADE) framework.

Results: Twenty RCTs were included, 17 included measures of fatigue $(n=1003)$ and 12 measures of sleep quality $(n=731)$. In comparison to usual care, exercise had beneficial effects on fatigue (Figure 1, $P<0.001$ ) but not on sleep quality (Figure 2, $P=0.06$ ). The most beneficial interventions for fatigue and improving sleep quality, were those in which fatigue was the primary outcome and those based on body-mind interventions, respectively (both, $\mathrm{P}<0.001$ ). A moderate risk of bias was present in most of the included studies.

Conclusion: According to the GRADE framework, this review provides low-to-moderate quality evidence that exercise is moderately effective for improving fatigue, and moderate evidence of no/meaningless effects of exercise to improve sleep quality. Further high quality RCTs are required to determine the effectiveness of exercise on fatigue, and in particular, sleep quality in fibromyalgia

\section{REFERENCES:}

[1] Macfarlane GJ, et al. Ann Rheum Dis 2017;76:318-28. Doi:10.1136/annrheumdis-2016-209724

[2] Bidonde J, et al. Cochrane Database Syst Rev 2017;2017. Jun 21;6: CD012700 doi:10.1002/14651858.CD012700

[3] Busch AJ, et al. Cochrane database Syst Rev 2013;:CD010884. doi:10.1002/14651858.CD010884

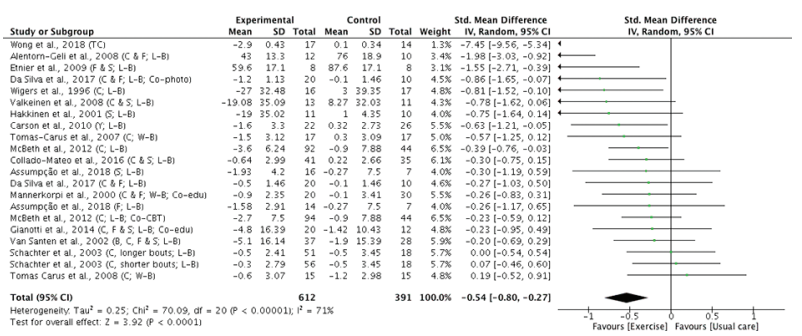

Figure 1. Pooled effects of randomised controlled trials analysing the effectiveness of physical exercise on reducing fatigue in people with fibromyalgia.

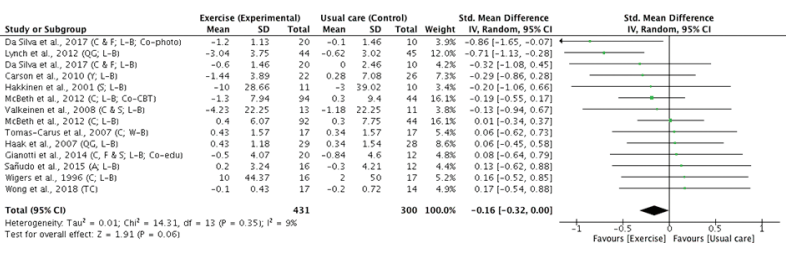

Figure 2. Pooled effects of randomised controlled trials analysing the effectiveness of physical exercise on enhancing sleep quality in people with fibromyalgia.

Analyses were conducted using a random effects model. $\mathrm{Cl}$, Confidence Interval; df, degrees of freedom; Std, Standardised; SD, Standard Deviation; IV, Inverse Variance; Co-, Co-intervention (Photo, phototherapy; edu, education); C, Cardiorespiratory exercise; F, flexibility exercise; S, Strength training; TC, Tai Chi; QG, Quigong; Y, Yoga; L- and W-B, land- and water-based exercise, respectively.

Acknowledgement: This study was funded by the Health and Social Care Public Health Agency, Northern Ireland (STL/5268/16) and the Spanish Ministry of Economy and Competitiveness (BES-2014-067612)

Disclosure of Interests: None declared

DOI: 10.1136/annrheumdis-2019-eular.4806

\section{FRI0711-HPR PATIENT PERSPECTIVES ON HOW TO IMPROVE MEDICATION EDUCATION}

Mirjam Hegeman, Jolanda Delsing, Jacqueline Luttikholt, Harald Vonkeman. Ziekenhuis Medisch Spectrum Twente, Rheumatology, Enschede, Netherlands

Background: EULAR recommends that people with inflammatory arthritis should have access to and be offered patient education throughout the course of their disease (1). The content and delivery of patient education should be individually tailored (2). Patients should be educated on their medication and know its purpose, mode of action, possible side effects and monitoring guidelines (3). Improving patients' ability to make informed choices and to use medication effectively and safely should result in significant benefits for the health service and improve patient well-being (4). Our institute's yearly Consumer Quality Index (CQI) revealed that our current medication education needs improvement.

Objectives: To improve medication education from the patient perspective. Methods: A representative cross-sectional sample of 100 rheumatoid arthritis patients was invited to complete a specially designed questionnaire, based on the Satisfaction with Information about Medicines Scale (SIMS) questionnaire (5). All caregivers at our Rheumatology unit (rheumatologists, trainees, rheumatology nurses) participated in single-blinded structured observational sessions of their regular patient consultation with the patient's consent. Data was collected on the type and content of medication information that was given during the consultation. Local ethical approval was obtained and patient confidentiality was assured.

Results: At present $40(40 \%)$ patients returned the questionnaire. Overall, patients are satisfied with the medication education provided (average overall satisfaction score of $7.4(0-10)$. However patients experienced insufficient education on the following topics;

- whether the medication has influence on sex life

- the risk of having side effects

- how to act when side effects occur

- possible interaction with concomitant medication

- whether the medication can cause drowsiness

Between December 2018 and January 2019 caregivers at our unit (4 rheumatologists, 2 trainees, 1 nurse) participated in multiple single-blinded observational sessions. In $100 \%$ of observed consultations medication information was provided. However, most caregivers did not address the topic of side effects during their consultation. Furthermore, in all cases caregivers failed to document in the electronic patient file which information was provided.

Conclusion: Rheumatoid arthritis patients express overall satisfaction with medication education but experience an unmet need for information on possible medication effects on their sex life, medication side effects and on interaction with concomitant medication. Further analysis of the questionnaires will be performed and a plan of improvement will be implemented to meet the patients need for more and better education on medication. Patient medication education will be implemented in a continuous cycle of improvement. 


\section{REFERENCES:}

[1] Zanghi HA, Ndosi M, Adams J, et al. (2015) EULAR recommendations for patients education for people with inflammatory arthritis.

[2] Daïen Cl, Hua C, Come B, et al. (2016) Pharmacologic and non-pharmacologic therapies in early arthritis: results of a systamatic literature informing the 2016 update of the EULAR recommendations for the management of early arthritis. RMD open 2016

[3] An Bord Altranais. (2003). Guidance to Nurses and Midwives on Medication Management. An Bord Altranais.

[4] Dublin.Roycroft-Malone J., Latter S., Yerrell P. and Shaw D. (2000). Nursing and medication education. Nursing Standard. 14. (50) 35-39.

[5] Horne R, Hankins M, Jenkins R. The Satisfaction with Information about Medicine Scale (SIMS): a new mesurement tool for audit and research. Qual Health Care. 2001;10:135-140

Disclosure of Interests: None declared

DOI: 10.1136/annrheumdis-2019-eular.7571

\section{FRI0712-HPR EFFICACY OF GRADED ACTIVITY WITH AND WITHOUT DAILY-MONITORED-WALKING ON SELECTED CLINICAL ATTRIBUTES OF PATIENTS WITH CONCOMITANT LOW- BACK PAIN AND TYPE-2 DIABETES}

Opeyemi Idowu' ${ }^{1}$, Ade Adeniyi'. ${ }^{1}$ University of Benin, Department of Physiotherapy, Benin City, Nigeria; ${ }^{2}$ University of Ibadan, Department of Physiotherapy, Ibadan, Nigeria

Background: Graded Activity (GA) is efficacious in managing clinical attributes of patients with Low-Back Pain (LBP) in the general population $[1,2]$. It is unknown whether $G A$ is efficacious in managing these clinical attributes in patients with concomitant LBP and Type-2 Diabetes (T2D) or additional daily-monitored-walking as a form of physical activity will be required.

Objectives: The objectives of this study were to investigating the effects of GA with and without daily-monitored-walking, and also to compare the efficacy of the two treatment modes on selected clinical attributes of patients with concomitant LBP and T2DM.

Methods: A single-blind controlled trial involving 58 patients (mean age: $48.3 \pm 9.4$ years, $64.7 \%$ females) with concomitant LBP and T2D who received treatment twice weekly for twelve weeks was conducted. Participants were randomized into GA or GA with daily-monitored-walking (GAMW) groups. Pain Intensity (PI), Back Extensors Endurance (BEE), Abdominal Muscular Endurance (AME) and Glycaemic Control (GC) was assessed using Visual Analogue Scale, Biering-Sorensen test, flexor endurance test and HBA1C analyser respectively at baseline, 4th, 8th and 12th week. Data were analysed using repeated measures ANOVA, medians, Mann-Whitney $U$ and Unpaired t-tests at $\alpha=0.05$.

Results: At baseline, treatment groups were not significantly different in their physical and clinical characteristics. There were within-group significant differences on $\mathrm{PI}, \mathrm{AME}$ and BEE in each of GA and GAMW groups across the time points of the study. Within group difference on GC was significant for GAMW $(6.3 \pm 0.9 \%, 5.7 \pm 0.7 \%)$ but not GA $(6.3 \pm 0.9 \%, 6.3$ $\pm 0.9 \%)$ groups. There were no significant difference between the effects of GA and GAMW on PI at week 4 (Median $=-0.78, I Q R=0.06$; Median=0.78, IQR=0.08), week $8 \quad$ (Median $=-1.72, \quad \mid \mathrm{QR}=0.06 ; \quad$ Median=-1.71, IQR=0.18) and week 12 (Median=-1.46, IQR=0.10; Median=-1.53, IQR $=0.18$ ) of the study. Further there was no significant difference between the effects of GA and GAMW on AME at weeks $4(6.64 \pm 0.15$; $6.65 \pm 0.09), 8(7.01 \pm 0.16 ; 7.03 \pm 0.1)$ and $12(5.16 \pm 0.05 ; 5.19 \pm 0.09)$ of the study. Graded activity with daily-monitored-walking had higher improvements than GA alone on BEE $(7.34 \pm 0.1,7.25 \pm 0.1)$ at week 8 , but not at week $4(2.37 \pm 0.08 ; 2.36 \pm 0.1)$ or $12(4.5 \pm 0.06 ; 4.53 \pm 0.08)$ of the study. The GAMW participants also had significant improvement on glycaemic control than GA participants $(-0.5 \pm 0.2 \%,-0.6 \pm 0.5 \%)$ at week 12 . Graded activity relieves back symptoms via the development of a sense of control over pain, elimination of pain avoidance as well as by improving overall physical fitness/function [3].

Conclusion: Graded activity with daily-monitored-walking produced positive effects on GC and yielded better improvement on BEE.

\section{REFERENCES:}

[1] Lindstrom, I., Ohlund, C., Eek, C., Wallin, L., Peterson, L., Fordyce, W.E. and Nachemson, A.L. 1992a. The effect of graded activity on patients with subacute low back pain: a randomized prospective clinical study with an operant-conditioning behavioural approach. Phys Ther. 72:279-293.

[2] Macedo LG, Latimer J, Maher CG, Hodges PW, McAuley JH, Nicholas MK, Tonin L, Stanton CJ, Stanton TR, Stafford R. Effect of motor control exercises versus graded activity in patients with chronic nonspecific low back pain: a randomized controlled trial. Phys Ther. 2012; 92:363-377.

[3] Bello Al, Quartey JNA, Lartey M. Efficacy of Behavioural Graded Activity Compared with Conventional Exercise Therapy in Chronic Nonspecific Low Back Pain: Implication for Direct Health Care Cost. Ghana Med J. 2015; 49:173-180.

Disclosure of Interests: None declared

DOI: 10.1136/annrheumdis-2019-eular.724

\section{FRI0713-HPR COMPARISON OF BIOPSYCHOSOCIAL STATUS OF PATIENTS WITH ANKYLOSING SPONDYLITIS WITH AND WITHOUT ANTI-TNF TREATMENT}

nur banu karaca ${ }^{1}$, Edibe Ünal ${ }^{1}$, Sedat Kiraz ${ }^{2} .{ }^{1}$ Hacettepe University Institution of Health Science, Faculty of Physical Therapy and Rehabilitation, Ankara, Turkey; ${ }^{2}$ Hacettepe University Faculty of Medicine, Department of Rheumatology, ankara, Turkey

Background: It is known that anti-TNF therapy is given to patients with persistently high disease activity despite conventional treatments according to the ASAS recommendations (1). Although biopsychosocial symptoms were well known, biopsychosocial assessments are insufficient in the literature.

Objectives: The aim of this study was to compare the biopsychosocial status between patients with AS who were decided to be treated with anti-TNF treatment and patients with AS who did not receive any antiTNF treatment. Also, it was aimed to investigate the effectiveness of 3 months anti-TNF treatment on biopsychosocial status.

Methods: 74 AS patients who are decided to receive anti-TNF treatment and 38 AS patients, who didn't, were included in the study. Socio-demographic informations of patients were collected. The mean age of the patients $(n=112)$ was $41,9 \pm 19,8$ years. Health Assessment Questionnaire (HAQ) was used to assess functional status and daily living activities. The Hospital Anxiety and Depression Scale (HADS) was used to assess anxiety and depression levels. Biopsychosocial status of the patients was evaluated by the BETY-Biopsychosocial Questionnaire (BETY-BQ) $(2,3)$. The same evaluations were repeated after 3 months in 36 patients using anti-TNF.

Results: A statistically significant difference was observed in BETY-BQ, HADS anxiety and HAQ scores, when the groups were compared. There was no statistically significant difference in HADS depression scores (Table 1). The difference after 3 months of anti-TNF treatment was significant in all parameters (Table 2).

FRI0713HPR Table 1. Difference between the patients who decided to receive anti-TNF and who did not.

\begin{tabular}{lccc}
\hline & $\begin{array}{c}\text { Anti-TNF Decided Group } \\
(\mathbf{n}=\mathbf{7 4})\end{array}$ & $\begin{array}{c}\text { Non-receiving Anti-TNF } \\
\text { Group } \\
(\mathbf{n = 3 8})\end{array}$ & $\begin{array}{c}\mathbf{p}- \\
\text { value }\end{array}$ \\
\hline HAQ & $14,33 \pm 10,1$ & $5,46 \pm 10,7$ &, $\mathbf{0 0 0}$ \\
HADS - Anxiety & $10,24 \pm 5,0$ & $7,58 \pm 5,0$ &, $\mathbf{0 1 1}$ \\
HADS - & $8,38 \pm 4,3$ & $6,45 \pm 4,3$ &, 053 \\
Depression & $59,32 \pm 24,1$ & &, 032 \\
BETY-BQ & & $46,74 \pm 25,8$ & \\
${ }^{*}$ Mann-Whitney U & & &
\end{tabular}

FRI0713HPR Table 2. First and 'After 3 months' measurements in patients who received anti-TNF treatment.

\begin{tabular}{lccr}
\hline $\mathbf{N}=\mathbf{3 6}$ & 1st measurement & 'After $\mathbf{3}$ months' measurement & p-value \\
\hline HAQ & $14,99 \pm 11,1$ & $5,36 \pm 10,6$ &, 000 \\
HADS - Anxiety & $10,33 \pm 5,3$ & $7,72 \pm 4,7$ &, 001 \\
HADS - Depression & $8,89 \pm 4,7$ & $5,78 \pm 4,7$ &, 000 \\
BETY-BQ & $59,33 \pm 25,1$ & $44,28 \pm 23,2$ &, 000
\end{tabular}

Wilcoxon Test

Conclusion: It was observed that the patients who were decided to be received anti-TNF treatment had worse functionality, anxiety, and biopsychosocial status than the patients who did not receive anti-TNF treatment. Anti-TNF treatment was found to be effective in three months period in terms of these biopsychosocial symptoms that the patients had.

\section{REFERENCES}

[1] Braun Jv, Van Den Berg R, Baraliakos X, Boehm H, Burgos-Vargas R, Collantes-Estevez E, et al. 2010 update of the ASAS/EULAR recommendations for the management of ankylosing spondylitis. Annals of the rheumatic diseases. 2011;70(6):896-904. 